\title{
Evaluation of the Relevant Features of Welding Defects in Radiographic Inspection
}

\author{
Antonio Alves de Carvalho*, Raphael Carlos de Sá Brito Suita, Romeu Ricardo da Silva, \\ João Marcos Alcoforado Rebello \\ Department of Metallurgical and Materials Engineering, Federal University of Rio de \\ Janeiro (UFRJ), C.P. 68505, 21945-970 Rio de Janeiro - RJ, Brazil
}

Received: December 20, 2002; Revised: May 12, 2003

\begin{abstract}
The use of X-ray as an inspection technique to ensure the integrity of industrial products dates from the beginning of the $20^{\text {th }}$ century. Therefore, it is a tool of non-destructive inspection widely known. Nowadays, however, there are several researches forward on the optimization of such inspection technique, mainly for the development of an automatic system of radiographic image analysis. That is, a system that can identify and classify the defects in the radiography. An important step in the construction of this system is the classification of defects, which is usually done by using some of their features. The purpose of this work is to study the relevance of some defect features in order to classify some of the main classes of defects. The employed technique is the linear correlation between the defect features and the classes of defects. A non-linear pattern classifier is used, implemented by a neural network, to evaluate the performance in the classification. The results showed the efficiency of the method used.
\end{abstract}

Keywords: nondestructive tests, radiography, welding defects, neural networks

\section{Introduction}

The radiography is a nondestructive method that uses the penetrating and ionizing inspection radiation to detect internal defects, which also detects, with high sensitivity, discontinuities with few millimeters of extension, when properly aligned with the incident beam. Mainly used in the petroleum, petrochemical, nuclear and power generation industries for the inspection of welds and castings especially, as well as in the war industry for the inspection of explosives, armaments and missiles, the radiography has played an important role in the quality assurance of the piece or component, in conformity with the requirements of the standards, specifications and codes of manufacturing. Besides, the industrial radiography plays a fundamental role in the quality documentation of the inspected product, because the projected image of the radiographic film represents the internal "photography" of the piece, which no other non-destructive test is able to show.

The reliable detection of defects is one of the most important tasks in non-destructive tests, mainly in the radiographic test, since the human factor still has a decisive influence on the evaluation of defects on the film. An incor- rect classification may disapprove a piece in good conditions or approve a piece with discontinuities exceeding the limit established by the applicable standards.

The purpose of the automation of the process of analysis of digitized radiography is to reduce the analysis time and eliminate the subjective aspect in the analysis done by the inspector, this way increasing the reliability in the inspection, considering that the automated evaluation is done by computer programs ${ }^{1-3}$. The automation of the analysis processes of radiography consists usually on the following steps: radiographic film digitizing, digital image processing, feature extractions of defects, and defect recognition through tools of pattern recognition ${ }^{1,4}$.

In this work, the authors describe a technique to evaluate the relevancy of some features to classify the main classes of weld defects on industrial radiographs. Furthermore, these features were used on a nonlinear pattern classifier as input vector to classify the weld defects: undercutting (UC), lack of penetration (LP), porosity (PO) and slag inclusion (SI). The nonlinear classifier were implemented by neural networks. The new results obtained by this work are the prove of the efficiency of the technique.

*e-mail: alves@metalmat.ufrj.br 


\section{Detection and Classification of Welding Defects on Radiographic Images}

The radiographic image processing is especially used to improve the image quality, making the analysis process easier, which consists of detecting and classifying defects on the film. In the conventional method, the analysis is done exclusively by the X-ray inspector. The progresses in computer science and the artificial intelligence techniques have allowed the defect classification to be carried out by using pattern recognition tools ${ }^{5,6}$, especially the methods that use artificial neural networks, which make the process automatic and more reliable, as it is not a subjective analysis. Pattern recognition are tasks routinely performed by humans. When someone who is driving a car looks at a traffic light, he/she is able to distinguish the information easily and start the proper procedure afterwards. When we observe a photograph, we can identify precisely the people in the photo even if we have seen them before for just a few times, although the people in the photo may wear different clothes, have different hair styles and be in different positions. These tasks, as many others, are simple for humans, but implementing them in a computer system is extremely complex ${ }^{7}$.

The techniques for pattern recognition implemented on radiographic digital image involve, mainly, the location and isolation of the objects (defects) on the radiographic image, and later, the defect identification (classification). The pattern recognition assumes that the image may have one or more defects, and that each defect belongs to a preset type, category or pattern class of defects. If a digitized image has sev-

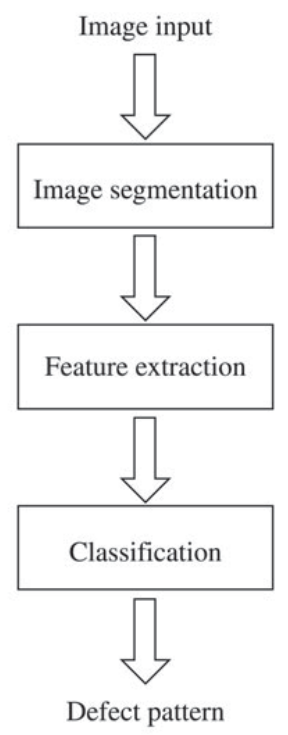

Figure 1. Step of the process of pattern recognition. eral defects, the process for pattern recognition consists of three main steps (Fig. 1) ${ }^{5}$, which are described as follows.

The first step is the image quality improvement or the defect isolation, in which each defect is located and isolated from the rest of the image. Several types of degradations and distortions, inherent in the processes of image acquisition, transmission and display contribute to limit the capacity to extract information. The main purpose of this image quality improvement step is to suppress or reduce the consequences of these barriers, making it easy to extract the relevant information. This improvement is usually done with the application of image digital filters ${ }^{5}$.

The second step is the extraction of defect features ${ }^{1}$, that is, where the defects are measured. One measurement is the value of any sizeable property of defect. A feature is a function of one or more measurements, which are registered in the computer to dimension any significant characteristic of the defect. This drastic reduction in the amount of information (if compared to the original image) represents all the knowledge on which the subsequent process of classification shall be based ${ }^{5}$. Therefore, the fewer features existing to represent a certain class, the fewer information will be processed. For example, if an intelligent system intends to classify banana and orange, only one feature of form is necessary to classify these two classes. However, the classes are not always so easily separated.

The use of features of defects is one of the mostly used techniques to classify the welding defects after their detection. In this case, the correct selection of the most relevant features in the identification of each class has a great importance in the recognition of such classes done by the intelligent system ${ }^{8}$. This selection is similar to the interpretation given by an inspector who, in most cases, recognizes first one type of welding defect in the radiography by visual characteristics, such as: location, shape, length, density (gray level), aspect ratio, etc., besides the welding conditions. So, an important study of the defect morphology at the image level is required to optimize the system performance ${ }^{8}$.

The third step of the pattern recognition process is the classification. This output is merely a decision considering the class that each defect belongs to. Each defect is considered to be an individual class, and the recognition is implemented as a process of classification. Each defect is directed to one of the several preset classes, which represent all possible types of defects expected to exist in the image.

\section{Analysis of the Linear Correlation Matrix}

The analysis of the linear correlation matrix of variables is well known in statistics ${ }^{9}$, being typically used to check the linear correlation between variables and, consequently, enable the reduction in the dimensionality of a multivariable data. The matrix elements are the linear correlation coeffi- 
cients, calculated by the equation (1):

$$
C(x, y)=\frac{1}{n} \sum_{i=1}^{n}\left(\frac{x_{i}-\bar{x}}{\sigma_{x}}\right)\left(\frac{y_{i}-\bar{y}}{\sigma_{y}}\right)
$$

where

$C(x, y)$ : linear correlation between variables $x$ and $y$.

$\bar{x}$ and $\bar{y}$ : mean values for variables $x$ and $y$ respectively.

$\sigma_{\mathrm{x}}$ and $\sigma_{\mathrm{y}}$ : standard deviations for variables $x$ and $y$ respectively.

The correlation $\mathrm{C}$ varies in the interval of $[-1,1]$. High values for the module $\mathrm{C}$, that is, $\mathrm{C}$ close to +1 or -1 , indicate that there is a great probability of a linear dependence between the two variables, and values for the module $\mathrm{C}$ of about zero indicate that there is a small probability of such dependence occurring.

If the two variables $x$ and $y$ are independent and random, certainly the expected value of the correlation will be zero. A method to evaluate the reliability in the existence or non-existence of correlation between the two variables described in Chatfield ${ }^{9}$ is to compare to the correlation of random variables: the correlation values that are higher than the ratio $2 / \sqrt{N}$, where $\mathrm{N}$ is the number of observations of the variables, indicate $95 \%$ of probability of variables being correlated, more details about this theory can be read on Chatfield's book 9 .

\section{Experimental Methodology}

\subsection{Image Digitizing and Processing}

Radiographic films of the IIW (International Institute of Welding)* patterns were digitized having primarily the classes of defects desired to be evaluated in this study: lack of penetration (LP), undercutting (UC), porosity (PO) and slag inclusion (SI). The radiographic images were digitized in a Flatbed UMAX Mirage II scanner, which operates in transmission and reflection mode, with resolution of 500 dpi (dots per inch) and gray scale at 256 levels ( 8 bits). The images were stored in TIFF format (with no compression). A microcomputer (PC type) and a image analysis program (Image Pro Plus 4.0 for Windows) were used.

After the image digitizing step, many program tools were analyzed to improve the image quality, and in general, a median-type low-pass filter was used to smooth the noise, and a high-pass filter or tools of contrast improvement were used to highlight the outlines of the defects. Some more details about this step can be find on the Silva's reference ${ }^{8}$. Mery ${ }^{1}$ also explains each step usually used on radiographic image enhancement. After this step, the image was calibrated and the defects identified on the image by visual way, as

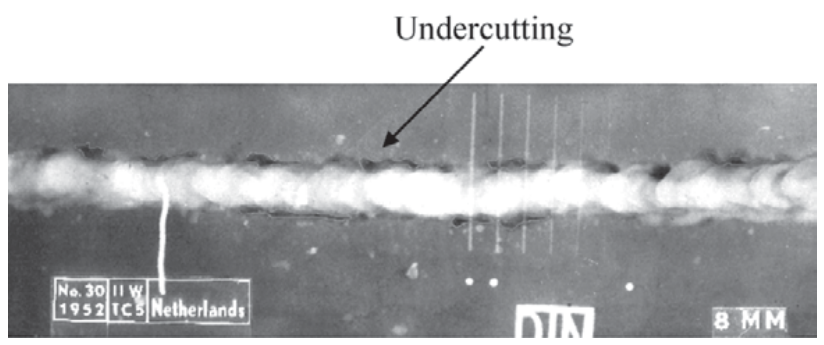

Figure 2. Identification of defects on the image.

shown on the Fig. 2. Once the defect was located, the features were calculated automatically by the software Image Pro Plus.

\subsection{Selecting and Defining the Features}

Eight features were selected for the classification of four classes of defects: lack of penetration (LP), undercutting (UC), porosity (PO) and slag inclusion (SI). The data used was composed of 29 lack of penetration observations and 30 observations including the remaining defect classes. A relatively high number of features were selected, with the purpose of showing the high relevance degree of some features to characterize certain classes of defects, which will be analyzed by the correlation study.

The adequate selection of features has fundamental importance for the subsequent classification process. Some criteria can make it easier to choose the best features. For example, the distance from the defect to the weld bead center distinguishes very well the undercutting from the lack of penetration, as the lack of penetration is in the weld bead center and the undercutting is usually at the edge ${ }^{8}$. However, the intention of this study is to evaluate the features that can provide satisfactory results with the automatic extraction.

Definition of the features used:

- Aspect Ratio (As): Ratios between the longest and the shortest axes of a equivalent ellipse within the defect area.

- Area/Box (AB): Ratio between the defect area and the area of the smallest rectangle that contains the defect.

- Box $X / Y(X / Y)$ : Ratio between the width and the height of the smallest rectangle that contains the defect.

- Angle (Ang): Angle between the shortest axis of the defect and the vertical line.

- Ratio between the radius $(R / r)$ : Ratio between the longest and the shortest radius of the defect.

- Roundness $(R)$ : It measures the ratio $P^{2} / 4 p A$, where ' $\mathrm{p}$ ' is the perimeter of the defect and ' $\mathrm{A}$ ' is the area.

- Fractal $(F)$ : Fractal dimension ${ }^{10}$ of the defect outline.

*Properly authorized by the IIW - International Institute of Welding. 

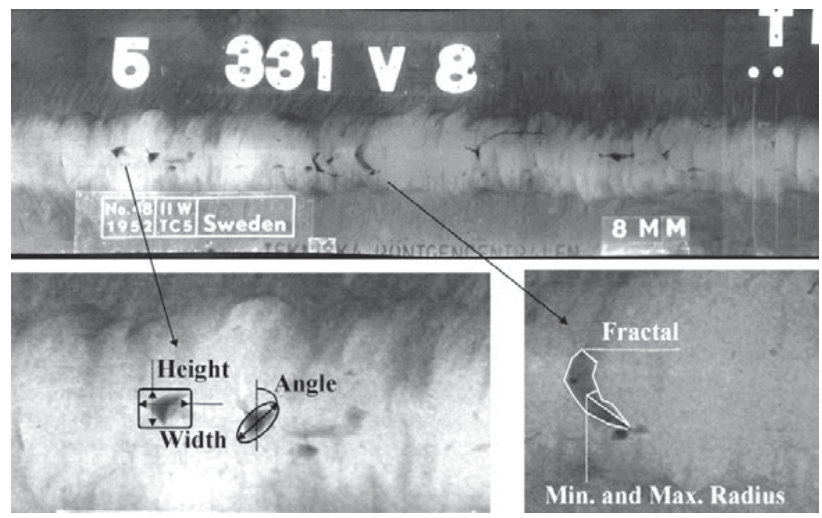

Figure 3. Example of one IIW pattern used and automatic extraction of the features.

- Medium Density (D): Medium density in gray tones of points from inside the defect.

Figure 3 shows a example of automatic extraction of the features done by the program Image Pro Plus:

\section{Results and Discussions}

In order to make the extracted data as representative as possible, not making the analysis improperly difficult, it is important to eliminate the outliers that are not included in the true representation of a defect class. Based on that, the measurements of the classes were adjusted to a standard normal distribution, and the atypical values that were not close to the average for more than three standard deviations were excluded from the data group, as shown on the Fig. 4.

Table 1 shows the values obtained from the correlation coefficients among the 8 features, and between the features and the 4 classes of defects analyzed in this study. This technique is used to evaluate the relevance of the features chosen for the classification of each defect class, which was calculated using the training set of the neural network ${ }^{11}$, so in this case, we have a matrix of twelve columns ( 8 features +4 classes). This technique is completely described on Silva $^{8}$. The criterion used to check the correlation existence follows the ratio $2 / \sqrt{N}$. As the total value of the features was 119 , so the values that were higher than $(0.18)$ indicate a probability of correlation over $95 \%$ (shaded cells) ${ }^{9}$. This table also shows the values of $2 / \sqrt{N}$ for each defect class. See as follows one analysis of the feature correlation for each defect class, based on Table 1.

- Lack of Penetration: The features R, AB and Ang indicate a correlation with this class, with the feature $\mathrm{R}$ showing the highest correlation.

- Undercutting: The features $\mathrm{AB}$ and $\mathrm{XY}$ display a high correlation rate with this class, followed by the features $\mathrm{AS}, \mathrm{R} / \mathrm{r}$ and $\mathrm{D}$.

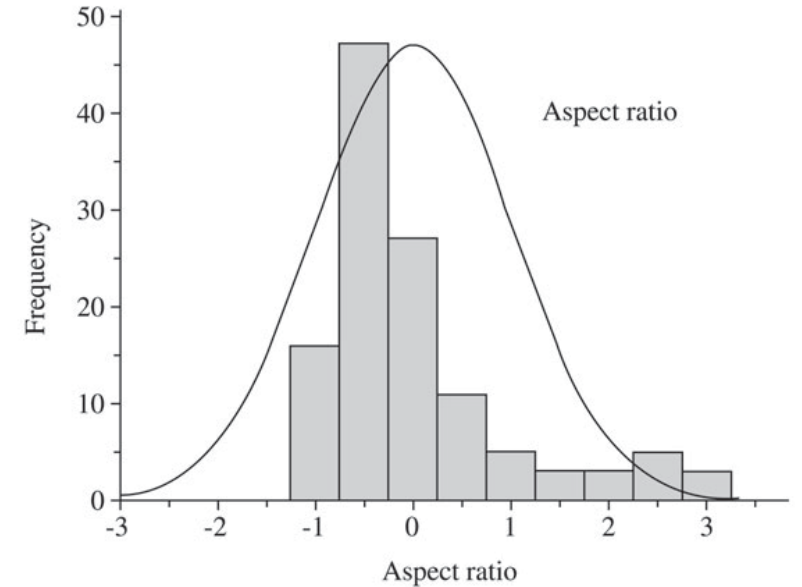

Figure 4. Distribution of frequency adjusted to the standard normal distribution.

- Porosity: The features R, R/r, AB and As indicate a correlation with this class, with the feature R showing the highest correlation.

- Slag Inclusion: The features Ang and R indicate a correlation with this defect class.

A nonlinear pattern classifier implemented with a backpropagation-type neural network ${ }^{6,11}$ is used with a hidden layer of 10 neurons and 4 neurons on the output layer ( 4 classes of defects), being the 8 features extracted from the radiographic image the inputs of the classifier ${ }^{11}$. By altering the number of neurons on the hidden layer and observing the training and performance error of the classifier at the end of the training, it is possible to obtain the best number of neurons on the hidden layer for the proposed study. By changing the number of neurons within the range of 7 to 15 on the hidden layer, the best result obtained (minimum error and maximum performance) was 10 neurons.

As mentioned before, the fewer features, the less computer efforts will be to classify the classes of defects. However, the system probability of not classifying the class or classifying it incorrectly might be higher, depending on the relevance of the features used.

The first step in the process to optimize the number of features was to use all of them in the classification and evaluate the performance of the classifier, which was $100 \%$ in this case.

In the second step, Table 1 was used to identify the features of higher relevance in the classification. It is possible to observe that the feature $\mathrm{AB}$ has relevance in the classification of the classes LP, UC and PO, while the feature Ang has relevance in the classification of the classes SI and LP. Then, it could be assumed that only these two features would be enough to classify the 4 classes of defects, but the evaluation of the classifier for these two features shows that the 
Table 1. Matrix of correlation with the correlated features.

\begin{tabular}{|c|c|c|c|c|c|c|c|c|c|c|c|c|}
\hline \multicolumn{9}{|c|}{ Features } & \multicolumn{4}{|c|}{ Defects } \\
\hline $2 / \sqrt{N}$ & & & & 0.18 & & & & & 0.37 & 0.36 & 0.36 & 0.36 \\
\hline & As & $\mathrm{AB}$ & $\mathrm{X} / \mathrm{Y}$ & Ang & $\mathrm{R} / \mathrm{r}$ & $\mathrm{R}$ & $\mathrm{F}$ & $\mathrm{D}$ & LP & UC & PO & SI \\
\hline As & 1 & & & & & & & & -0.133 & 0.492 & -0.377 & 0.017 \\
\hline $\mathrm{AB}$ & -0.406 & 1 & & & & & & & 0.440 & -0.757 & 0.440 & -0.118 \\
\hline $\mathrm{X} / \mathrm{Y}$ & 0.493 & -0.528 & 1 & & & & & & -0.226 & 0.696 & -0.258 & -0.214 \\
\hline Ang & 0.140 & -0.001 & 0.278 & 1 & & & & & 0.407 & 0.225 & -0.241 & -0.387 \\
\hline $\mathrm{R} / \mathrm{r}$ & 0.393 & -0.340 & 0.288 & 0.152 & 1 & & & & -0.188 & 0.396 & -0.362 & 0.152 \\
\hline $\mathrm{R}$ & 0.131 & -0.232 & 0.322 & 0.468 & -0.021 & 1 & & & 0.537 & 0.321 & -0.475 & -0.377 \\
\hline F & 0.034 & 0.082 & 0.070 & 0.049 & 0.092 & 0.060 & 1 & & 0.049 & 0.006 & 0.049 & -0.104 \\
\hline $\mathrm{D}$ & -0.214 & 0.278 & -0.185 & -0.108 & -0.286 & -0.058 & 0.050 & 1 & 0.152 & -0.361 & 0.259 & -0.049 \\
\hline
\end{tabular}

performance obtained was $87.5 \%$, which is a consistent result, considering the small amount of information supplied to the classifier.

After that, in order to improve the classification performance, another feature was added to the classifier. As Table 1 shows, the feature $\mathrm{R}$ has relevance in the classification of the classes LP, PO and SI. So, once again the classifier is evaluated, but now with $\mathrm{AB}$, Ang and $\mathrm{R}$ as the input data. The result obtained in this case was $93.33 \%$, increasing significantly the performance of the classification system. Keeping the same idea, one more feature $(\mathrm{R} / \mathrm{r})$ was added to the classifier, as it has relevance to classify the classes $\mathrm{UC}$ and PO. With the features AB, Ang, R and R/r, the performance increased, reaching $97.5 \%$. Giving continuity to the evaluation, the feature As was included, and the performance was also $97.5 \%$. Then, it can be concluded that the feature As does not add any relevant information. So, it was decided to remove the feature $\mathrm{R} / \mathrm{r}$, as the feature As classifies the same classes as the feature $\mathrm{R} / \mathrm{r}$. In this case, the performance of the classifier with the features $\mathrm{As}, \mathrm{AB}$, Ang and $\mathrm{R}$ was $99.16 \%$, that is, approximately the same performance obtained with the 8 features. So, it follows that evidently what really matters is the "quality" of the features used, that is, the relevance of the features in the classification of the defect classes here studied. The obtained result is excellent, because it provided the reduction in the data dimension of $50 \%$ for a performance of about $100 \%$ in the classification of the classes here studied. It is important to point out that the dimensionality reduction in multivariate systems and the intelligent use of data constitute in one of the most studied areas nowadays in pattern recognition.

It is also used to evaluate the nonlinear classifier with an test set (an input vector that was not used at the training step), because it is important to evaluate the generalization capability of the neural network. It was not possible at this work as there were not sufficient data.
An analysis can also be done by using the correlation between one feature and another. Thus, if two features are strongly correlated, the exclusion of either feature probably will not affect the classification significantly, as there might be a large amount of redundant information being provided to the classifier. This fact could be confirmed with the features As and $\mathrm{R} / \mathrm{r}$, as the exclusion of $\mathrm{R} / \mathrm{r}$ did not affect the classification. This technique has already been analyzed by $\mathrm{Silva}^{8}$ applied to other features, but new features were investigated in this study, presenting important results that can be used in a pattern recognition system.

There are other techniques that can be applied to reduce the input dimension of the classifier and evaluate the relevancy of each feature. A technique, which is also implemented by neural network, is described by Seixas ${ }^{12}$.

Extraction of features from radiographic images is not an easy task, since errors may occur in the measurement process. So, the question that arises is to find out what is the best cost-benefit ratio, that is, how to obtain the best precision rate to meet the requirements of the problem in question as practically as possible.

\section{Conclusions}

The technique of linear correlation between one feature and another and between features and classes of defects showed that it is really possible to reduce the dimensionality of the data set used, because there is a higher relevance of some features to classify certain classes and the linear correlation between one feature and another shows redundancy of information being offered to the classifier.

The results obtained in this work confirmed the others results which were published by Silva ${ }^{8}$, however, in this work, some different features were obtained by automatic extraction. The main conclusion is that it is possible to project a system of classification of welding defects using a reduced number of relevant features. 


\section{Acknowledgements}

Authors wish to acknowledge CNPq, CAPES and FAPERJ for the financial support and scholarships and the International Institute of Welding for permission given to publish the present study using the radiographic standards.

\section{References}

1. Mery, D. Processing Digital X-ray Images and Its Applications in the Automated Visual Inspection of Aluminum Casting, $3^{\text {rd }}$ Pan American Conference for Nondestructive Testing - PANNDT, Rio de Janeiro RJ, June 2003.

2. Liao, T.W.; Tang, K. Automated Extraction of Welds from Digitized Radiographic Images Based on MLP Neural Networks. Applied Artificial Intelligence, v. 11, p. 197-218, 1997.

3. Kato, Y.; Okumura, T.; Matsui, S. et al. Development of an Automatic Weld Defect Identification System for Radiographic Testing. Welding in the Word, v. 30, n. 7-8, p. 182-188, 1992.

4. Silva, R.R. Radiographic Image Processing and Analysis of Defects in Weld Bead. Master's Degree. PEMM/
COPPE/UFRJ. RJ, Brazil,1999. (In Portuguese).

5. Gonzalez, R.C.; Woods, R.E. Digital Image Processing, $1^{\text {a }}$ edição, USA, Addison-Wesley Publishing Company, 1992.

6. Bishop, C.M. Neural Networks for Pattern Recognition. New York, Oxford University Press, 1995.

7. Siqueira, M.H.S. Inspeção em Grandes Distâncias Utilizando Ondas Ultra-Sônicas Guiadas e Inteligência Artificial, Tese D.Sc., PEMM/COPPE/UFRJ, Rio de Janeiro, Janeiro, 2002.

8. Silva, R.R.; Calôba, L.P.; Siqueira, M.H.S. et al., Evaluation of the Relevant Characteristic Parameters of Welding Defects and Probability of Correct Classification Using Linear Classifiers, Insight, v. 44, n. 10, Outubro, 2002.

9. Chatfield, C.; Collins, A.J. Introduction to Multivariate Analysis, Chapman and Hall, USA, 1980.

10. Mandelbrot, B.B. Fractal Geometry of Nature, W.H. Freeman and Co., New York, 1983.

11. Haykin, S. Neural Networks, A Comprehensive Foundation, Macmillian College Publishing. Inc., 1994.

12. Seixas, J.M.; Calôba, L.P.; Delpino, I. Relevance Criteria for Variance Selection in Classifier Designs. In: International Conference on Engineering Applications of Neural Networks, p. 451-454, 1996. 